\title{
Pattern formation during air injection into granular materials confined in a circular Hele-Shaw cell
}

\author{
Ø. Johnsen, ${ }^{1}$ R. Toussaint, ${ }^{1,2}$ K. J. Måløy, ${ }^{1}$ and E. G. Flekkøy ${ }^{1}$ \\ ${ }^{1}$ Department of Physics, University of Oslo, P.O. Box 1048 Blindern, 0316 Oslo, Norway \\ ${ }^{2}$ IPGS University Louis Pasteur of Strasbourg, France \\ (Received 23 October 2005; published 11 July 2006)
}

\begin{abstract}
We investigate the dynamics of granular materials confined in a radial Hele-Shaw cell, during central air injection. The behavior of this granular system, driven by its interstitial fluid, is studied both experimentally and numerically. This allows us to explore the associated pattern formation process, characterize its features and dynamics. We classify different hydrodynamic regimes as function of the injection pressure. The numerical model takes into account the interactions between the granular material and the interstitial fluid, as well as the solid-solid interactions between the grains and the confining plates. Numerical and experimental results are comparable, both to reproduce the hydrodynamical regimes experimentally observed, as well as the dynamical features associated to fingering and compacting.
\end{abstract}

DOI: 10.1103/PhysRevE.74.011301

PACS number(s): 81.05.Rm, 47.11.-j, 83.50. $-\mathrm{v}$

\section{INTRODUCTION}

We have studied pattern formations during air injection in a granular material confined in a radial Hele-Shaw cell [1] and have characterized and quantified the features of the granular pattern formations and the associated dynamic processes.

The dry porous matrix is loosely packed inside the cell, and the particles are able to move together with the fluid phase. The origin of the instability is similar to what was described by Saffmann and Taylor [2]. The pressure gradient in the fluid is the driving force of the granular motion. The pressure is more or less constant within a central grainless region, and the driving pressure gradient is largest at the tip of the longest finger of this structure. According to Darcy's law [3], the fluid flow and the fluid drag is highest at the tips, so that the growth of the most advanced finger is favored at the expense of the rest of the structure. The stabilizing mechanism, on the other hand, is not surface tension as in the classical Saffmann-Taylor system, but friction mobilized between the granular material and the confining plates. The resulting patterns observed are either uniform, almost circularly shaped patterns or branched finger patterns, depending on the injection pressure.

We are dealing with a coupled fluid-grain flow where the grains are small and gas-grain interactions are of central importance. Such interactions play a key role in a wide range of systems from fluidized beds [4-7] to flow in tubes [8-13], and ticking hour glasses [14,15].

A system similar to ours, wherein a fluid invades a confined granular material, was studied experimentally by van Damme et al. [16] in 1993. In contrast to that study, ours involves quantitative measurements and comparison with simulations. In the situation wherein a fluid is retracted from a confined nonconsolidated porous medium, several hydrodynamic regimes have also been reported $[17,18]$.

Other systems with general similarities and important geological aspects to it include those of multiphase flow in porous media [19-39]. The pattern formations and instabilities that we observe in our system bear resemblance to those seen in Hele-Shaw cell experiments using both Newtonian [2,40-42] and non-Newtonian fluids [43-46]. In the nonNewtonian case a transition from a viscous fingering regime to a viscoelastic fracturing regime $[47,48]$ has been observed. The difference between these studies and the present one is that our structures depend entirely on the existence of static and sliding friction.

Like the viscoelastic fracture experiments our system can provide insight into the underlying physics of geological hydrofracture processes [49-53]. However, while fracture in viscoelastic media involves separated fluids, the experiment involve interpenetrating phases, and there is an effect of pressure diffusion of the air through the grain packing that has no analog in the viscoelastic systems. Unlike our system of nonconsolidated grains, viscoelastic fracturing and hydraulic fracturing of solids have been previously studied by numerical simulations [54-57].

This article is organized as follows: in Sec. II, the experimental model, setup, and sample preparation are described. In Sec. III, we give a classification of the observed pressure regimes and discuss the underlying physics. In Sec. IV, we present our simulation model and basic equations used in the algorithm. In Sec. V, the classification of the different regimes is recovered in the simulations. We then turn to quantitative measures on our system in Sec. VI before concluding and summarizing in Sec. VII.

\section{SYSTEM DESCRIPTION}

The system consists of a horizontally fixed circular HeleShaw cell [1] $0.45 \mathrm{~m}$ in diameter, separated with $1 \mathrm{~mm}$ ball bearing beads, and then clamped together at three points. The injection hole is $6 \mathrm{~mm}$ in diameter. The granular material, consists of polydisperse spherical glass beads, with diameters between $75-150 \mu \mathrm{m}$. Beads of this size are sensitive to the humidity of the ambient air; excessive humidity creates capillary bridges between the particles making the material more cohesive, while at a too low humidity the electrostatic forces make them stick together. The relative humidity in the 


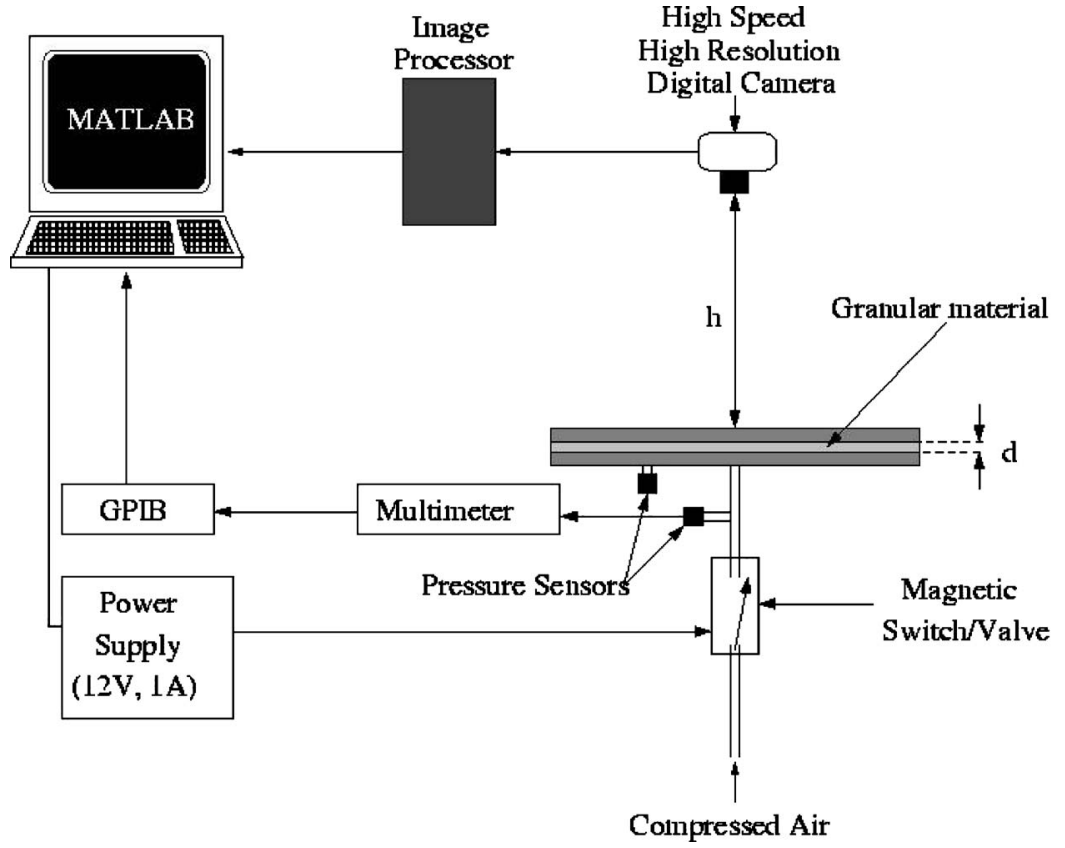

FIG. 1. Experimental setup: The horizontally fixed Hele-Shaw cell is connected to a pressure source. A high-speed, high-resolution CCD camera is used to follow the development of the displacement pattern and the pressure is read at the inlet and in the middle of the sample. Air is injected at constant overpressure in the center of the cell when the magnetic valve is triggered to open, and a displacement pattern appears. room is therefore controlled at approximately 30\% using a humidifier/dehumidifier.

Filling the Hele-Shaw cell homogeneously with powder is not an easy and straightforward task, and might be realized in many different ways. In order to have a loosely packed granular layer as homogeneous as possible, and to optimize the reproducibility of the initial state, we have developed the following filling procedure: We keep the cell in an upright position, close the bottom boundary, and let the particles rain down continuously over the system width using a guide, instead of filling from a point, which gives texture of different density due to avalanches around the angle of repose. The particles are packed under gravity when held vertically and the Janssen effect contributes to making the granular packing density homogeneous [58].

The cell is placed in a horizontal position after it is filled, the side boundary is opened, and the air inlet is connected to a gravitationally driven pressure control device. Two Honeywell 26PCAFA6G pressure sensors records the pressure at the inlet and in the middle of the cell. At the same time as the pressure recording is started, images are taken every $1 / 250 \mathrm{~s}$ using a Redlake MotionPro high-speed CCD camera with spatial resolution of $1280 \times 1280$. Central injection of air into the model is triggered by switching a magnetic valve open, $1 \mathrm{~s}$ after the image and the pressure recording is initiated. Figure 1 shows a simple sketch of the experimental setup.

\section{CLASSIFICATION OF OBSERVED REGIMES}

\section{A. Phenomenology}

The characteristics of the pattern formation changes with the injection pressure. At threshold values for the injection pressure there are sharp transitions from one situation to the other. By tuning the injection pressure in small steps, we find pressure thresholds that separate four pressure regimes.

\section{The first pressure regime: $\Delta P<P_{1}$}

When the injection pressure $\Delta P$ is smaller than $P_{1}$ no pattern will form in the granular material. The pressure is not high enough to displace particles, so that the injected air just permeates through the pores of the granular layer. Below this threshold we define the first pressure regime. Given the conditions for our experimental configuration, $P_{1} \approx 0.20 \mathrm{kPa}$ will be the lower threshold for pattern formation.

Note that although the granular velocity is zero in this situation, the fluid velocity is finite. As can be established by flux measurements or by pressure measurements and by use of the Darcy law, the typical air velocity decays radially from around $30 \mathrm{~mm} \mathrm{~s}^{-1}$ along the injection zone to $0.4 \mathrm{~mm} \mathrm{~s}^{-1}$ close to the outer boundary, for $\Delta P \sim P_{1}$.

\section{The second pressure regime: $P_{1}<\Delta P<P_{2}$}

For injection pressures $P_{1}<\Delta P<P_{2}$, defined as the second pressure regime, the particles are pushed outwards, leaving a relatively small and quite circular pattern with no grains, as shown in Fig. 2. Experimentally, we find the lower threshold for pattern formation to be $P_{1} \approx 0.20 \mathrm{kPa}$ and the upper threshold for this regime $P_{2}=1.90 \pm 0.1 \mathrm{kPa}$. The emptied structure is typically stabilized after $\sim 0.1 \mathrm{~s}$. The size of the structure increases with increasing pressure.

As the emptied structure grows, the material ahead of the structure compactifies over a depth dependent on the size of the structure. With sufficiently high spatial resolution, this zone can be found using image analysis. By subtracting, pixel by pixel, the image at a given time from an image taken before gas injection, we can detect minute motions of grains. By thresholding this subtraction image, there is a welldefined densely speckled region, like a halo, around the emptied zone. By performing noise averaging on the difference image, we can detect the front of the compacted region. An example of this front is inserted onto the image from the experiment in Fig. 2. 


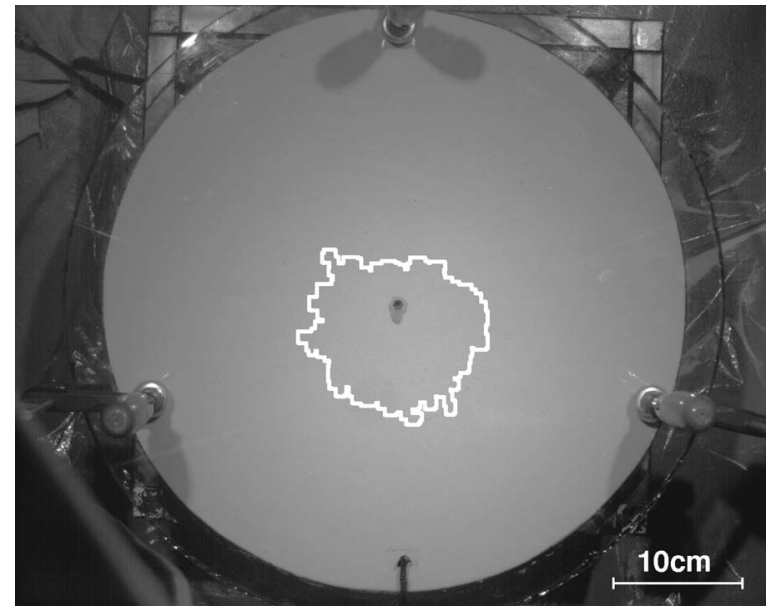

FIG. 2. A picture of a fully developed structure within the second pressure regime at injection pressure $\Delta P=1.26 \mathrm{kPa}$. Ahead of the emptied region there is a zone where the material is compacted, shown by the white line.

The more or less circular uniform structures, which are empty of grains in the second regime, are observed up to $\Delta P \approx 2.00 \mathrm{kPa}$, at which point the structure becomes unstable.

This circular symmetry breaking is quantitatively identified as follows: The boundary of the final empty region has radial coordinates bounded by $r_{\min }$ and $r_{\max }$. When $r_{\max } / r_{\min }>2$, the pattern is classified as characteristic of the third pressure regime.

\section{The third pressure regime: $P_{2}<\Delta P<P_{3}$}

Between $P_{2}$ and $P_{3}$, an instability in the displacement process becomes significant, and a finger pattern very similar to those seen for invasion in non-Newtonian fluids starts developing (cf. Fig. 3). We find $P_{2}=1.90 \pm 0.1 \mathrm{kPa}$ and $P_{3}$ $=2.40 \pm 0.1 \mathrm{kPa}$. The structure typically ceases to grow and stabilizes by $\sim 0.1 \mathrm{~s}$ after initiation. The instability over the front appears as branched fingers. The number of fingers at a given pressure is variable among different experiments. This can be attributed to a strong sensitivity to the details of the initial state. However, a noticeable trend is that the structure develops more in the direction along which the particles were poured into the cell.

During filling, the cell is held vertically and the particles are packed under gravity, which results in the friction between the granular layer and the confining plates being polarized in the opposite direction of the gravity field [58]. Correspondingly, there will be an anisotropy in the granular stress, which may explain the direction of the displacement pattern.

As the cell is filled the weight on the bottom particles also becomes slightly higher than the case is at the top, resulting in a slightly higher density and higher normal stress acting on the confining plates. Particles in the upper part of the cell may therefore be pushed and compacted more easily. This might also contribute to a preferred directionality opposite to gravity during filling, though the Janssen effect [58] will restrict the influence of this feature. (a)

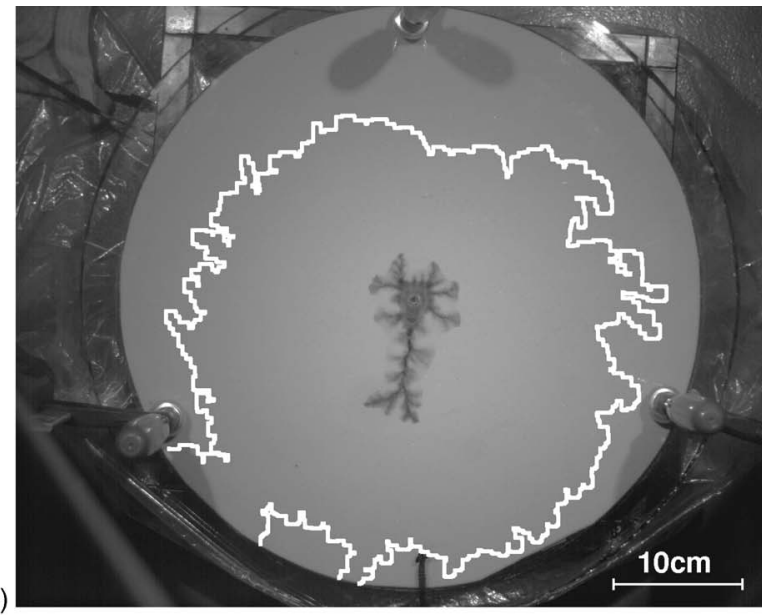

(b)

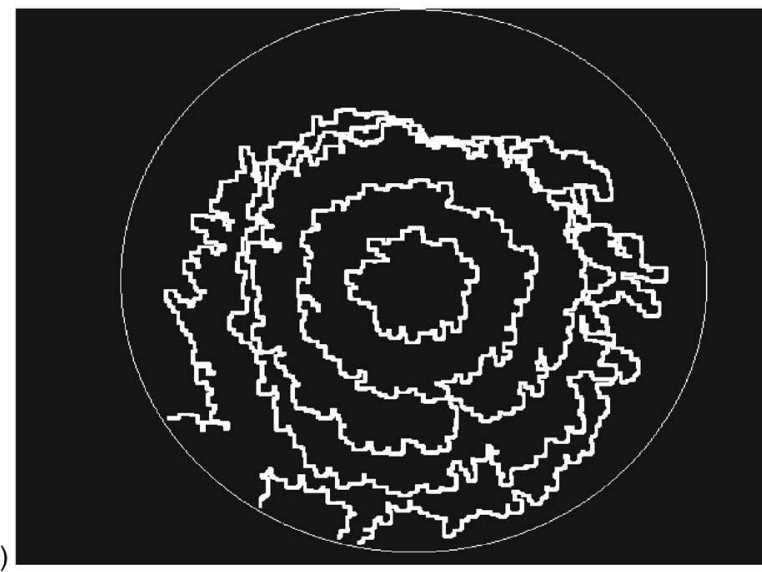

FIG. 3. (a) Displacement pattern at $\Delta P=2.34 \mathrm{kPa}$, in the third pressure regime. The front of the compacted region is indicated with a white colored line. The short black line at the bottom of the image shows the direction of the particle motion during filling of the cell. (b) The front of the compacted region at $t=0.008 \mathrm{~s}, t$ $=0.020 \mathrm{~s}, t=0.080 \mathrm{~s}, t=0.480 \mathrm{~s}$, and $t=0.800 \mathrm{~s}$ after injection.

When increasing the pressure, the empty and compacted structures get bigger. We follow the development of the compacted front in Fig. 3(b). The radial velocity of the front is large immediately after injection starts, but the growth rate of the emptied structure decreases due to the increasing in-plane friction.

There is a crossover between regimes 2 and 3 for pressures $1.80 \mathrm{kPa}<\Delta P<2.00 \mathrm{kPa}$. In this pressure interval one usually obtain a circular structure, but sometimes a branched structure. For pressures above $2.00 \mathrm{kPa}$, on the other hand, unstable structures are always observed, and we therefore use this value to define the crossover. We believe that this crossover has to do with the sample preparations where the granular packing cannot be duplicated from experiment to experiment.

Ahead of the longest finger, the compacted region may reach all the way up to the open boundary before it stops evolving. When this is the case, we are close to the border between the third and the fourth pressure regime. 


\section{The fourth pressure regime: $\Delta P>P_{3}$}

If we exceed $P_{3}$, which in our case is $P_{3}=2.40 \pm 0.1 \mathrm{kPa}$, the empty finger formation breaks through the granular packing, creating an eroded channel from the inlet to the external boundary.

A finger structure similar to those seen for the higher pressures in the third regime forms in the order of a tenth of a second. The front slows down a little before the most advanced finger accelerates toward breakthrough of the external boundary. As it accelerates, the air flow erodes the interior of the advancing finger. When we have a finger leading from the injection point to the open boundary, the pressure within the emptied region cannot be maintained with this pressure source, and then the rest of the structure will not be able to grow any further. The breakthrough finger "chooses" the direction where the particles were poured into the cell. At very high pressures, typically $\sim 4.00 \mathrm{kPa}$ and higher, it is not unusual to have more than one finger breaking through the granular material.

Between pressure regimes 3 and 4 , in the interval $2.30 \mathrm{kPa}<\Delta P<2.50 \mathrm{kPa}$, we occasionally have a finger breaking through, but this is not systematic.

\section{B. Physical discussion}

The origin of the instability is similar to what was described by Saffmann and Taylor [2]. The pressure gradient, $\nabla P$ is the driving force of the granular motion and the pressure field in the system is described by Darcy's law [3]. Within the fingers, the permeability is huge compared to the one in the pore space of the granular material, which will favor air flow in the emptied region. The pressure is more or less constant within the emptied structure and the driving pressure gradient $\nabla P=\Delta P / l$ is largest at the tip of the longest finger since here the distance $l$ from the tip to the open boundary is the smallest. Growth of the most advanced finger is therefore favored at the expense of the rest of the structure.

As the granular bulk is pushed upon, particles are displaced and rearranged in such a way that the granular material is compacted in a zone around the emptied structure, as indicated in Figs. 2-4. When granular motion is induced, the in-plane forces on the grains will cause a granular pressure to act in the transverse directions as well, as is illustrated in Fig. 5. The "induced" granular force acting perpendicular on the upper plate will be denoted as $P_{g}^{\perp}$. On the bottom plate there will also be a rather small contribution from gravity, $\rho g d$, where $g$ is gravity, $d$ the plate spacing, and $\rho$ is the mass density of the porous medium, i.e., $\rho=\rho_{s} \rho_{g}$ with $\rho_{g}$ the mass density of the particles themselves, and $\rho_{s}$ the local solid fraction.

The mobilized friction force per unit surface is described by a Coulomb friction model, i.e., it opposes the granular motion, and it is bounded by a Coulomb friction coefficient $\gamma$, times the normal pressure exerted by the grains. The friction force exerted by the top and bottom plate are respectively denoted $F_{\gamma}^{1}$ and $F_{\gamma}^{2}$, and the total friction force exerted by both plates over the local granular column is $F_{\gamma}=F_{\gamma}^{1}$ $+F_{\gamma}^{2}$. The normal pressure exerted against the top plate is $P_{g}^{\perp}$,

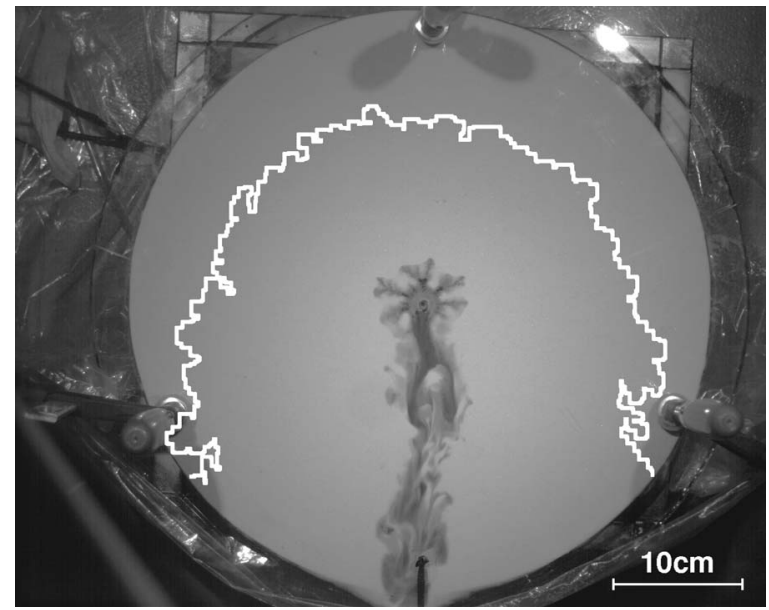

FIG. 4. Injection pressure $\Delta P=2.58 \mathrm{kPa}$ is within the fourth pressure regime where the structure breaks through the model. In this frame the finger has reached the outer boundary. The front of the compacted region is indicated with the white line.

and the one exerted against the bottom plate also accounts for the weight of the local granular column, i.e., $\left(P_{g}^{\perp}+\rho g d\right)$. The normal pressure $P_{g}^{\perp}$ itself arises from the compression of the granular matrix. It is considered to be proportional to the in-plane pressure acting on the grains from the surrounding grains in contact, $P_{g}^{\|}$, with a proportionality constant $\lambda / 2$ : this amounts to assume locally a Janssen [58] hypothesis for the grains between the confining plates, as illustrated in Fig. 5. Thus, we assume that $P_{g}^{\perp}=\lambda P_{g}^{\|} / 2$, and that the friction exerted by the top and bottom plates are, respectively, $F_{\gamma}^{1}$ $\leqslant \gamma \lambda P_{g}^{\|} / 2$ and $F_{\gamma}^{2} \leqslant \gamma\left[\lambda P_{g}^{\|} / 2+\rho g d\right]$, so that the total friction exerted by the confining plates per unit area is

$$
F \leqslant \gamma P_{g}^{\perp}=\gamma\left(\lambda P_{g}^{\|}+\rho g d\right) .
$$

In other words, we assume that much of the stress acting upon the area of the displacement front is transmitted to the glass plates through particle contacts, in a way similar to Janssen's silo experiments [58]. Friction is the stabilizing phenomenon in our system.

As the injected air flows through the granular material, the pressure gradient will smoothen out and be reduced over the front. When the friction force is large enough to balance the

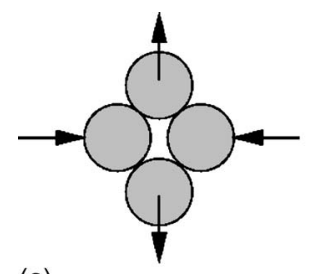

(a)

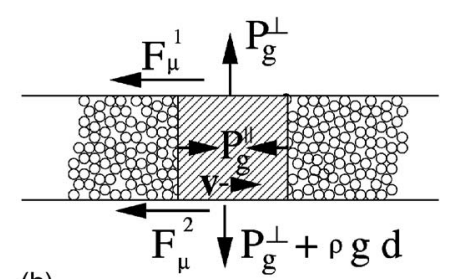

(b)
FIG. 5. (a) A sketch of the principle of how a vertical force is induced when applying a horizontal force on particles in contact. In our case the granular layer is about ten particle diameters. (b) The forces in action, where $F_{\gamma}$ is the friction force exerted by the plates on the material, $\rho g d$ the column weight, and $P_{g}^{\perp}$ and $P_{g}^{\|}$a normal and in-plane stress, respectively. 

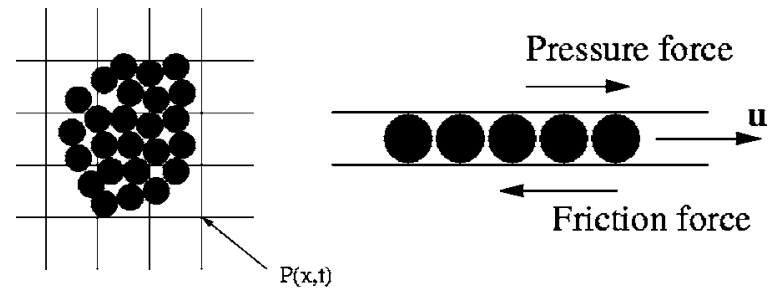

Friction force

A

B

FIG. 6. A conceptual picture of the model. (A) The top view shows the particles and the grid onto which the pressure equation is discretized. (B) The side view shows how the granular layer in the experiment is represented by a single layer in the simulations, and it shows the action of the friction forces and the pressure forces.

hydrodynamic drag on the particles, the emptied structure will stop developing. How these processes are cast in a mathematical description is the subject of the next section.

\section{SIMULATION MODEL}

\section{A. Principles}

Figure 6 illustrates conceptually the basis for the model. The gas flow takes place between grains that define a local permeability $\kappa$.

The details of the model are presented in Ref. [7]. In the following, we sketch its main features and the particular adaptation to the present application. The main assumption of the model is that the fluid dynamics may be described on a spatial scale above the grain scale, and that the fluid may then be described adequately in terms of the local pressure only. The solid phase, which is simulated in terms of individual particles, defines a deformable porous medium through which the gas flows.

The fluid pressure satisfies the equation

$$
\phi\left(\frac{\partial P}{\partial t}+\mathbf{u} \cdot \nabla P\right)=\nabla \cdot\left(P \frac{\kappa}{\mu} \nabla P\right)-P \nabla \cdot \mathbf{u},
$$

where $\phi$ is the local porosity, $\mathbf{u}$ the local granular velocity, $\mu$ the viscosity of air, and $\kappa$ the permeability.

In all the experiments, the flow velocity of the air is below $5 \mathrm{~cm} / \mathrm{s}$, so that the Reynolds number based on particle diameter is less than 0.2. In this flow regime, we are justified in using the Carman-Kozeny relation for the permeability as long as the porosity is smaller than roughly 0.7. See Ref. [59] for a more elaborate discussion of continuum equations such as Eq. (2).

Writing the permeability in terms of the local solid volume fraction $\left(\rho_{s}=1-\phi\right)$, the Carman-Kozeny relation [60] takes the form

$$
\kappa\left(\rho_{s}\right)=\frac{a^{2}}{45} \frac{\left(1-\rho_{s}\right)^{3}}{\rho_{s}^{2}},
$$

where $a$ is the particle radius.

The particles evolve according to Newton's second law:

$$
m \frac{d \mathbf{v}}{d t}=m \mathbf{g}+\mathbf{F}_{I}-\frac{\nabla P}{\rho_{n}}+\mathbf{F}_{\gamma}
$$

where $\mathbf{g}$ is the gravity, $m$ the particle mass, $\mathbf{F}_{I}$ the interparticle force, $\mathbf{F}_{\gamma}$ is the friction force of Eq. (1), and $\rho_{n}$ $=\rho_{s} \rho_{g} / m$ is the number density, with $\rho_{g}$ the mass density of the material composing the particles.

The repulsive interaction force $F_{I}$ between two particles in contacts is modeled as a frictionless linear elastic contact, i.e., its direction lies along the separation vector between the particle centers, and its magnitude is proportional to the overlap distance between the particles. The linear repulsion model used here is meant to be a numerical approximation of a hard-sphere model. The proportionality factor between force and overlap, or repulsive spring constant, is set such as the characteristic overlap between the particles is a negligible fraction of their distance in the simulations. In other terms, the characteristic time associated with a particle-particle collision is much smaller than any other elementary characteristic times in this model (which are a particle advection time due to the applied overpressure, another one due to gravity, and a characteristic time of diffusion of the pressure field at particle scale). We have checked numerically that changing this proportionality constant by a factor of 5 did not affect the simulation results; i.e., that we indeed effectively model a hard-sphere situation.

In order to model systems of size comparable to the experimental ones, we need to use grains of effective size larger than the experimental ones; i.e., numerical grains representing clusters of real grains. Indeed, the experiments performed involve flows of roughly $10^{8}$ grains, which is beyond numerical capacities for the model described here. Thus, we chose to model numerical particles of diameter equal to the plate separation. The simulations are thus performed using a quasi-two-dimensional (2D) granular code, in order to model a situation whereing typically $10-15$ grains lie in the thickness of the system. This is justified a priori by the fact that when motion happens in the experiments, the full granular layer is displaced. Note, however, that in order to evaluate the granular/fluid interactions, the permeability evaluation is based on the real size of the pores; i.e., on the diameter of the real grains. In addition, an important three-dimensional feature of the granular population is incorporated in the numerical model, by evaluating a three-dimensional Cauchy stress tensor in order to incorporate friction with the confining plates, as we will detail hereafter.

To compute the friction force $F_{\gamma}$ exerted by the plates via a Janssen hypothesis from Eq. (1), the average in-plane stress exerted over a particle of index $a$, in contact with a set of particles $b \in C_{a}$, is obtained after [61-63], as

$$
\begin{aligned}
\bar{\sigma}_{i j}^{\|} & =\left(1 / V_{a}\right) \int_{V_{a}} \sigma_{i j} d V \\
& =\left(1 / V_{a}\right) \sum_{b \in C_{a}} x_{i}^{b} f_{j}^{b}
\end{aligned}
$$

where $x^{b}$ is the position of the contact with the grain $b, f^{b}$ the contact force exerted by the grain $b$ over the grain of index $a$, 
$V_{a}$ is the grain volume, and the magnitude of the in-plane stress is $P_{a}^{\|}=-\left(\bar{\sigma}_{11}+\bar{\sigma}_{22}\right) / 2$.

In this application, we carry out the simulations in 2D and use molecular dynamics integrated with the velocity Verlet scheme to solve Eq. (4) [64]. Soft-sphere molecular dynamics [65] and contact dynamics [66] could be used instead.

For the model to work in practice, it is necessary to introduce a cutoff $\rho_{\text {min }}$ on the density. This has to do with limitations both of the Carman-Kozeny relation and the present numerics (see [7] for a more detailed discussion of this point). This cutoff is implemented as follows: Whenever the measured solid fraction $\rho_{s}$ is lower than $\rho_{\min }=0.25$, it is replaced by the cutoff $\rho_{s}=\rho_{\min }$ in the evaluation of the permeability by Carman-Kozeny [Eq. (3)].

Although the practical implementation of the present model in three dimensions is not significantly harder than in two dimensions, we wish to simulate a two-dimensional system because it is numerically less expensive. However, the Carman-Kozeny equation (3) is a three-dimensional relation as it gives the permeability in terms of the volume fraction of spheres $\rho_{s}$, and we wish in the end to compare our results to real three-dimensional experiments. Consequently, we need to transform the area fraction of grains in the simulations $\rho_{s}^{(2 \mathrm{D})}$, to the volume fraction $\rho_{s}$ in such a way that the close packed value of $\rho_{s}^{(2 \mathrm{D})}$ corresponds to the close packed value of $\rho_{s}$. Such linear transform of $\rho_{s}$, mapping 0 onto 0 , and the close packed value of $\rho_{s}^{(2 \mathrm{D})}$ onto its counterpart for the threedimensional problem, is approximately achieved by the transformation $\rho_{s}=(2 / 3) \rho_{s}^{(2 D)}$, which we use in the following $[7,67,68]$.

\section{B. Preparation of the initial stage}

Since the behavior of a granular material is strongly dependent on its history of deformation [69], and since the dynamical behavior of our system, and the initial stress state over the system, is sensitive on the initial preparation procedure [70], special attention is devoted to this initial state in order to match as closely as possible the experimental situation. As in the experiments, particles are initially stacked under gravity in a vertical cell. Particles are launched one by one from random positions over a flat bottom surface, they fall vertically and topple off already formed piles until they reach a geometrically stable position [cf. Fig. 7(a)]. Particles are considered as hard spheres without friction in the contact in this stage, and the collisions are totally inelastic; i.e., the particle velocity is set to zero after each contact. This entirely determines the geometrical stacking rule. Since friction is neglected in this preparation stage, this procedure builds a classical isostatic packing of polydisperse hard spheres. This defines a geometrical rule to stack the particles. In order to avoid the formation of hexagonally ordered crystallites of large extent arising from an artificially monodisperse particle size distribution in a two-dimensional packing, we use polydisperse particles. The granulometry used corresponds to a flat distribution of diameters, chosen such as the relative dispersion of the diameters of the numerical particles matches the one of the experiments; i.e., with a factor of 2 between the upper and lower cutoff of the granulometry.

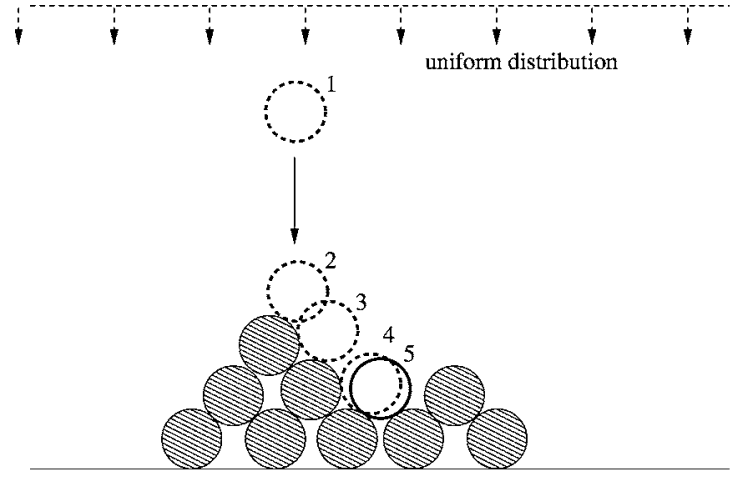

(a) Geometrical stacking under gravity

from a uniform grain-by-grain raining distribution

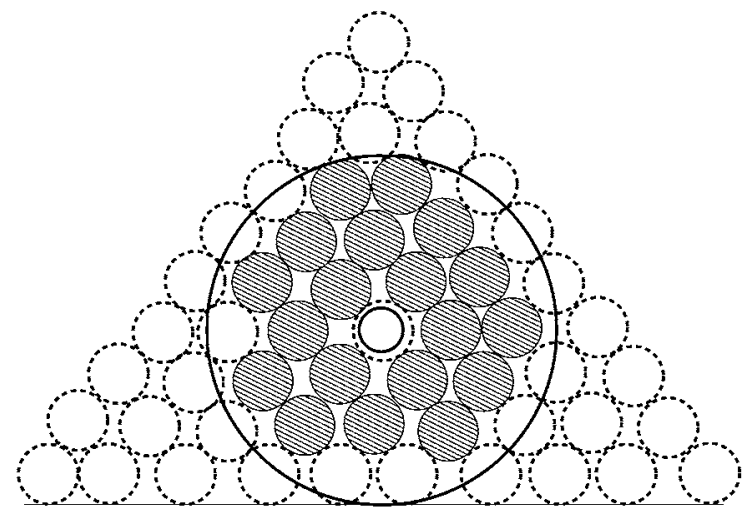

(b) removal of the grains outside the radial cell cache

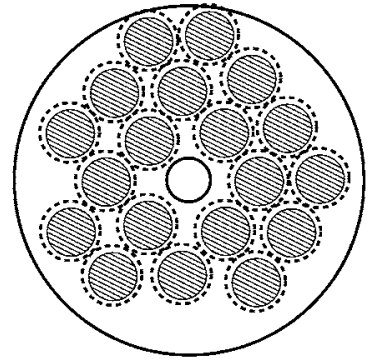

(c) normalization of the grain radii to achieve a given average porosity.

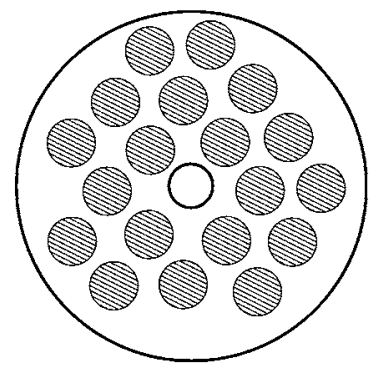

(d) resulting initial stage for the central injection simulation

FIG. 7. The preparation stage. (a) The grains are stacked one by one over a basic plane, as hard spheres piling up under gravity, with purely inelastic collisions and no friction between them. (b) Grains outside a circular cell with a central hole are removed from the ensemble. (c) The diameter of the grains is reduced by an overall factor to produce a prescribed initial average density. (d) Initial state for the injection stage. 
In a second stage, once a pile is built, we remove the particles that are not entirely in the radial cell, which has a crown shape limited by a central hole and an external circular boundary [cf. Fig. 7(b)]. This mimics the second step of the experimental preparation procedure: in experiments, particles were first stacked under gravity with the help of a guiding channel leading to the vertically placed cell, closed at the bottom, up to complete filling of the system. Next, the cell was placed horizontally, and the guides and plug of the central hole were removed, leading to the removal of the particles outside the outer radius.

Eventually, a third numerical preparation stage is added to fine tune the initial porosity so that the deviation in solid fraction from the random close packed value is the same in simulations and experiments. More precisely, if $\rho_{0}^{\exp }$ and $\rho_{c}^{\exp }$ are the initial and closed packed densities in the experiments, respectively, and $\rho_{0}^{(2 \mathrm{D})}$ and $\rho_{c}^{(2 \mathrm{D})}$ are the initial and close packed densities in the simulations, respectively, we impose the equality

$$
\frac{\rho_{c}^{(2 \mathrm{D})}-\rho_{0}^{(2 \mathrm{D})}}{\rho_{c}^{(2 \mathrm{D})}}=\frac{\rho_{c}^{\exp }-\rho_{0}^{\exp }}{\rho_{c}^{\exp }} .
$$

This is achieved by slightly reducing the particle radii in the simulations prior to air injection [see Fig. 7(c)]. Initially, the $2 \mathrm{D}$ packing fraction is $\simeq 0.75$, which is slightly below the known 2D random close packing fraction value of 0.84 [67]. The measured deviation is $\left(\rho_{c}^{\exp }-\rho_{0}^{\exp }\right) / \rho_{c}^{\exp } \approx 0.032$, as will be detailed in Sec. VI.

Alternatively, simulations can be performed without the last shrinking stage in the packing preparation. Such packing preparation only utilizes gravity, as in the experiments. However, since the model is two dimensional, the resulting packing fraction is different from the experimental one. Notably, the two-dimensional state resulting from the sole gravitational stacking is closer to a limiting close packing fraction than the three-dimensional experimental one. As a result, simulations carried from such alternative initial state leads to a smaller ratio of the central empty zone size over the compact zone size, i.e., without this fine-tuning stage, the simulated central empty zones are smaller, all other things kept equal. The other structural features described in Sec. VI and hydrodynamic regimes as a function of pressure, are otherwise unaffected by this last preparation stage. The only reason to include this optional third shrinking stage is to fine tune the size of the central empty fingers in order to better match the experiments.

There are eventually two remaining free parameters in such models, corresponding in Eq. (1) to $\lambda$, the Janssen proportionality parameter between in plane and normal stress, and $\gamma$, the Coulomb friction coefficient between the particles and the confining plates. These have been chosen to match as closely as possible:

1. The classification of regimes as function of the applied overpressure, i.e., the fact that four pressure regimes are observed, with transition pressures $P_{1}, P_{2}$, and $P_{3}$, corresponding to the experimental ones, up to roughly $30 \%$.

2. The time to breakthrough, where a given internal finger reaches the outer boundary, which was required to lie within $30 \%$ of the experimental one in the simulations carried out.

Exploring the parameter space of these two remaining parameters, to satisfy such constraints, led to the use of parameters $\lambda=1$ and $\gamma=0.25$ in the simulations.

\section{NUMERICAL ASPECTS: OBSERVED REGIMES AS FUNCTION OF THE PRESSURE}

We will here show how the numerical simulations render for the observed phenomenology in the experiments.

\section{A. Low injection pressure}

As in the experiments, there is a lower threshold for the imposed central pressure below which no granular motion takes place, and for which there is only air permeation through the material, held in place by friction exerted by the confining plates. For imposed pressures sufficient to exit this trivial regime, the same mechanism is observed for moderate imposed central pressures: In Fig. 8, we display the pressure field and the grain positions for a simulation carried out with an overpressure of $2 \mathrm{kPa}$. The color code ranges from green for atmospheric pressure, to red for overpressures exceeding $1 \mathrm{kPa}$ (half of the imposed central one). First, the pressure field diffuses through the granular material, and quickly adapts to the initial configuration of the granular packing [cf. Fig. 8(b), $15 \mathrm{~ms}$ after initial time]. The drag created by the pressure gradient is sufficient to overcome friction, and the fluid flow evacuates the granular material from the center, forming an empty central zone. This central motion compacts the material over a growing depth ahead of the central decompacted zone (cf. Fig. 9). In this figure, the color code represents the local permeability of the granular packing, which is evaluated from the Carman Kozeny expression [Eq. (3)]. The permeability gives the diffusion constant $D$ $=P \kappa / \mu$ in the fluid flow equations.

As the material is compacted ahead of the emptied zone, the friction against the plates is mobilized increasingly, and this leads to the stabilization of the granular motion. In this simulation, the granular motion is stabilized in Figs. 8 and 9(d), after $60 \mathrm{~ms}$. In this stationary state, the mobilized friction forces exerted by the side plates, balances exactly the fluid drag exerted by the air that flows permanently between the grains, driven by the pressure gradient shown in Fig. $8(\mathrm{~d})$.

To make a quantitative comparison with the experiments, we have adopted in these simulations, measures of a central emptied area $A_{i}$, and of the compacted area $A_{c}$, that were inspired by the experimental image analysis techniques: $A_{i}$ is the area that is left in the center after the grains have moved, which is connected to the central hole, after subtraction of the area of the initial central hole. $A_{c}$ is the area of the zones where the solid fraction has increased by more than $2 \%$ relatively to the initial solid fraction. Both definitions are illustrated on Fig. 10, $15 \mathrm{~ms}$ after the start of the injection. These areas will be used in Sec. VI to compare the dynamics of the simulations and experiments. 
(a)
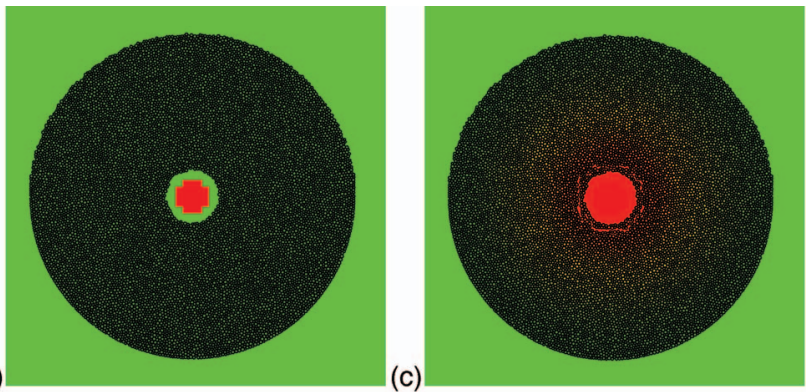

(b)

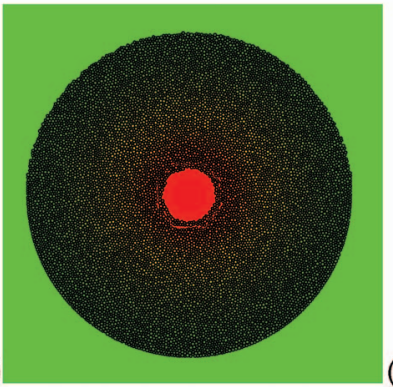

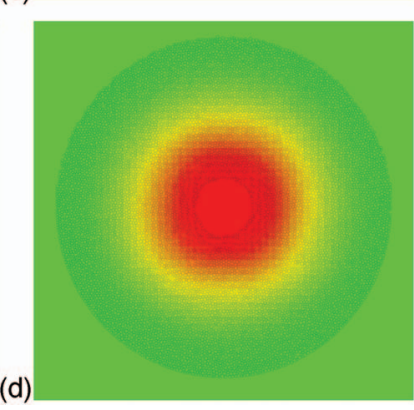

FIG. 8. (Color) Low injection pressure regime. The grains are represented in black, the color code of the background represents the overpressure: from green at zero overpressure, to red for overpressures reaching or exceeding half of the central one. (a)-(d) correspond, respectively, to times $0,15,45$, and $60 \mathrm{~ms}$ after the central overpressure is imposed. The decompaction of a central zone is observed, similarly to the experiments. The system stabilizes in state (d). Note that air still flows in this stable stationary state, as shows the nonhomogeneous pressure field displayed through transparent grains in (d).

\section{B. Higher injection pressure: breakthrough regime}

As in the experiments, the increase of the injection pressure leads to other type of flow behaviors. As the pressure is increased, two characteristic features are observed: the roughly circular symmetry of the patterns observed at lower pressures breaks down, and the finger formation is observed. The next characteristic feature is the formation of a large empty finger breaking through the compacted granular material. These features are illustrated in Figs. 11 and 12, which correspond to snapshots of the pressure field and permeability field in a simulation carried at an imposed central overpressure of $3 \mathrm{kPa}$.

The preparation procedure, Coulomb friction parameter $\gamma$ and Janssen proportionality parameter $\lambda$ are identical to those of the previous simulations.

Figure 11 illustrates that the pressure field mainly adapts to the shape of the granular material, in a system of such dimensions: As shown in Fig. 11(b), the characteristic response time of the pressure field to the shape of the overall granular material is initially much shorter than the response time of the granular material itself to the imposed global pressure difference across the system. However, both times become comparable in the latest stages of the simulation, where a large empty finger breaks through.

Another interesting feature of these simulations is that in addition to the central empty zones, the compacted area ahead, and the finger formation, another type of structure

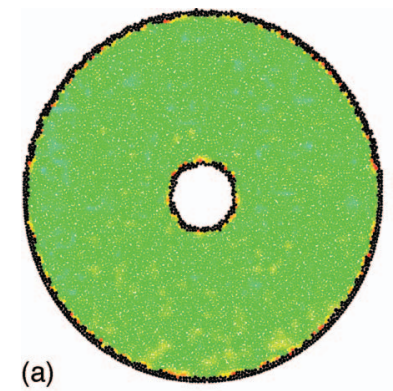

(a)

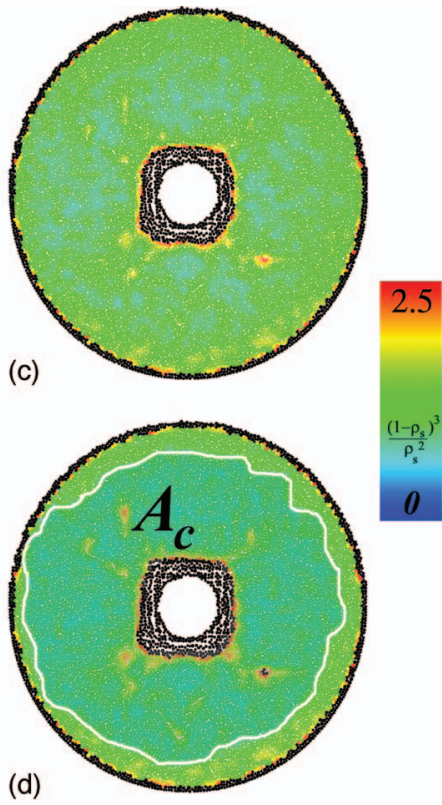

(b)

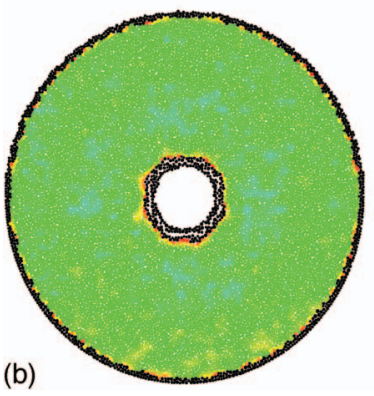

(d)

FIG. 9. (Color) Permeability representation of Fig. 8. The color code represents the local permeability, and thereby the solid fraction. Blue corresponds to $\kappa=0$, red to a two-dimensional solid fraction of 0.5. Larger local permeabilities are represented with black grains. The color bar represents $\rho_{s}^{3} /\left(1-\rho_{s}\right)^{2}$, which is proportional to the permeability $\kappa$. This quantity goes from 0 (blue) to 2.5 (red). In (d), the limit of the compacted region in the ultimate stationary state is displayed by a white line.

arises: Close to the external boundary in Figs. 12(c) and 12(d), bubbles start to form. The formation of these isolated empty zones is reminiscent of the granular bubble formation in some fluidized beds $[11,13]$, and it is fundamentally linked to the granular nature of our system; i.e., similar patterns are never formed by immiscible fluids that do not interpenetrate. The bubbles progress outwards towards the external open boundary of the system. The nucleation zone where such bubbles appear, grows during time, and its internal boundary progresses inwards, as is indicated in Figs. 12(b)-12(e). This illustrates a decompaction mechanism starting from the outer boundary, and allows the system to unjam. Note that contrary to the compaction mechanism starting from the center, which is mediated by solid contacts between grains, this decompaction mechanism is mediated essentially by the fluid-solid coupling.
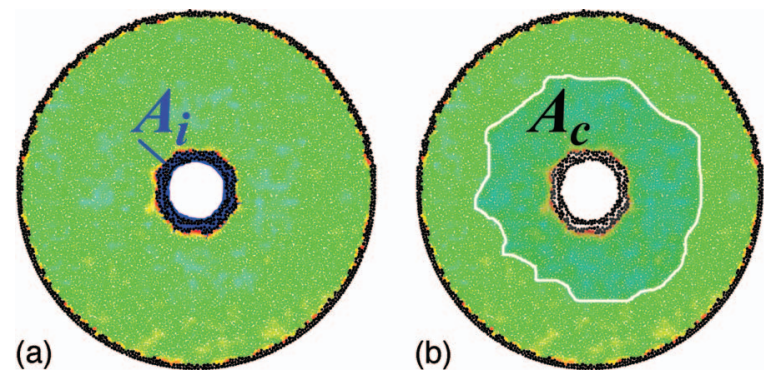

FIG. 10. (Color) (a) Central emptied area $A_{i}$, in blue, versus (b) compacted area $A_{c}$, after $15 \mathrm{~ms}$ of injection in the same lowpressure simulation 
(a)
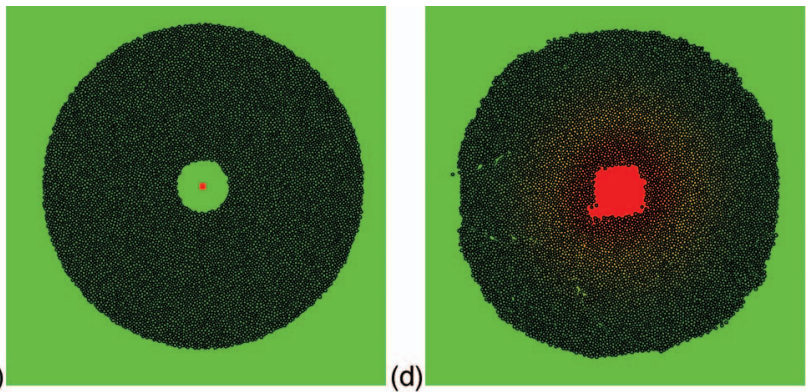

(b)
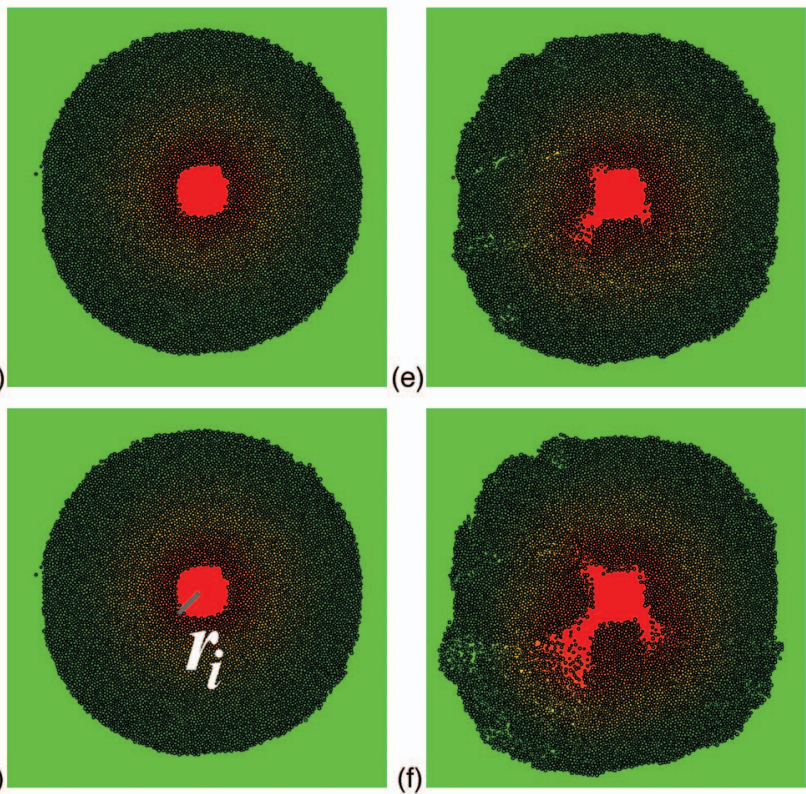

FIG. 11. (Color) Simulation snapshots at an imposed overpressure of $3 \mathrm{kPa}$. The color code in the background of the grains represents the pressure field and is the same as in Fig. 8. Times are (a) $0 \mathrm{~s}$, (b) $40 \mathrm{~ms}$, (c) $80 \mathrm{~ms}$, (d) $120 \mathrm{~ms}$, (e) $200 \mathrm{~ms}$, and (f) $280 \mathrm{~ms}$

To establish a quantitative comparison with the experiments in Sec. VI (cf. Fig. 14), we define measures of the radii of three types of structures, inspired by the experimental techniques: $r_{i}$, shown in Fig. 11(c), is the maximum radial coordinate of the geometrically connected central zone empty of grains. The radius $r_{c}$, shown in Figs. 12(c) and $12(\mathrm{~d})$, is the maximum radial coordinate of the zone geometrically connected to the center, by regions where the locally averaged two-dimensional porosity exceeds 0.49 ; i.e., the zone that includes grains represented in black in Figs. 11 and 12. Eventually, $r_{d}$, shown in Figs. 12(c) and 12(d), represents the minimum radial coordinate of the bubble zone, or, to be more precise, the decompaction area, where the solid fraction is lower than 0.5 (black color code) and still unconnected to the central zone. Note in Figs. 11(c) and 11(d) that while $r_{i}$ and $r_{c}$ are growing functions of time, $r_{d}$ decreases.

Although such definitions and choice of threshold for the solid fraction are necessarily subjective, they have the advantage of allowing a quantitative comparison. However, some unavoidable discrepancies are naturally expected between numerical and experimental measures of the areas of these three types of zones (central finger, compacted zone, decompacted zone).

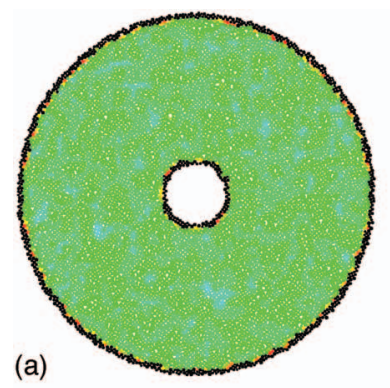

(d)
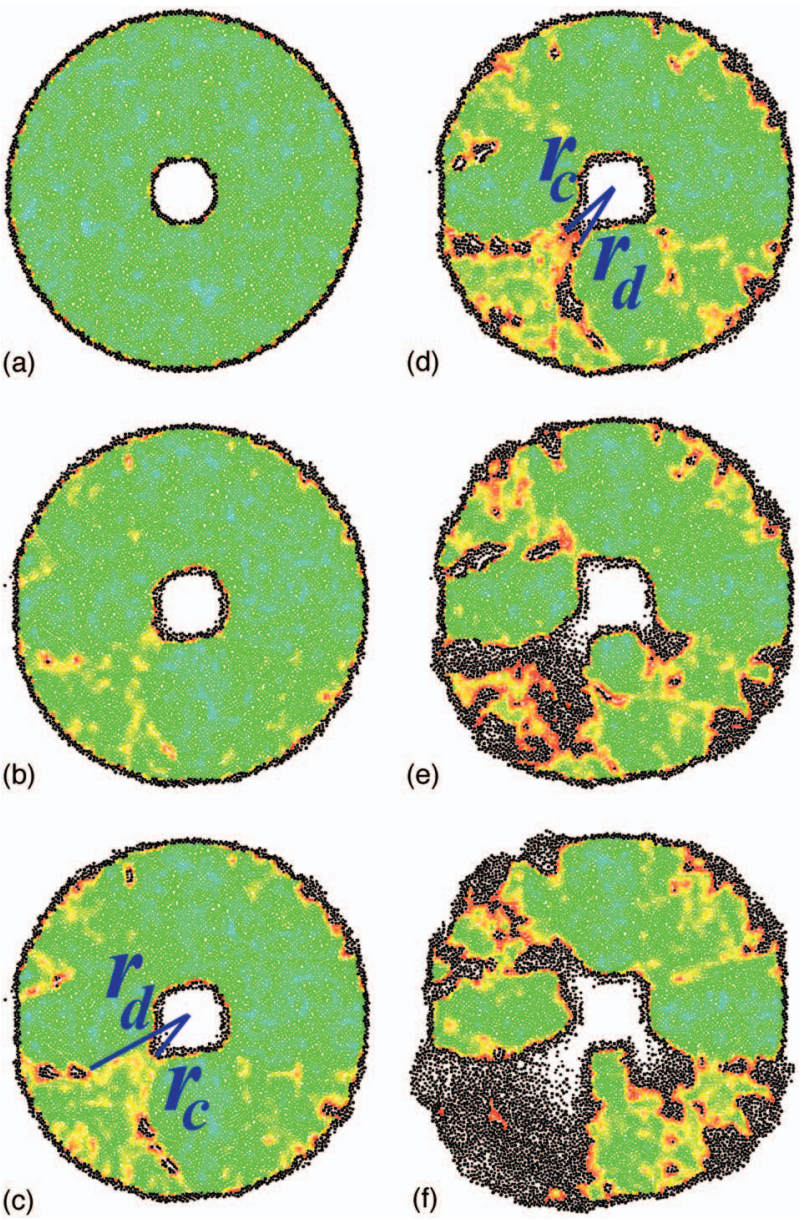

FIG. 12. (Color) Permeability map associated with the same snapshots as in Fig. 11. The radii $r_{i}, r_{c}$ and $r_{d}$ refer to the radii of the central empty zone, the zone ahead with a large enough motion so that $\phi>0.5$, and the decompactification zone progressing inwards from the external boundary.

\section{DYNAMICAL ASPECTS}

In Fig. 13, $r_{i}$ is followed in time for three experiments. We see that for the case of injection pressures within pressure regimes 2 and 3 , in this case $\Delta P=1.78 \mathrm{kPa}$ and $\Delta P$ $=2.20 \mathrm{kPa}$, the pattern grows rapidly in the beginning up to $\sim 0.1 \mathrm{~s}$ From $\sim 0.1 \mathrm{~s}$ the growth rate is reduced due to mobilization of friction. The structures fully saturates after $\sim 0.4 \mathrm{~s}$. In this particular example, the pattern formations reaches final radial size $\sim 1.7 \mathrm{~cm}$ after $0.44 \mathrm{~s}$ for the system in pressure regime 2 , and $\sim 4.9 \mathrm{~cm}$ after $0.43 \mathrm{~s}$ for the system in pressure regime 3.

The growth characteristics is similar for the compacted region in Fig. 13(b). After an initial stage where the zone is established it grows to a plateau within $\sim 0.1 \mathrm{~s}$. There is some small expansion of the compacted region within this plateau matching the motion of the emptied structure.

At higher pressures, $\Delta P=2.58 \mathrm{kPa}$, belonging to the fourth pressure regime the mobilized friction slows down the development and speed of the growing structure. As the emptied structure continues to develop, the material ahead of this structure compacts over a larger area. This is a manifes- 

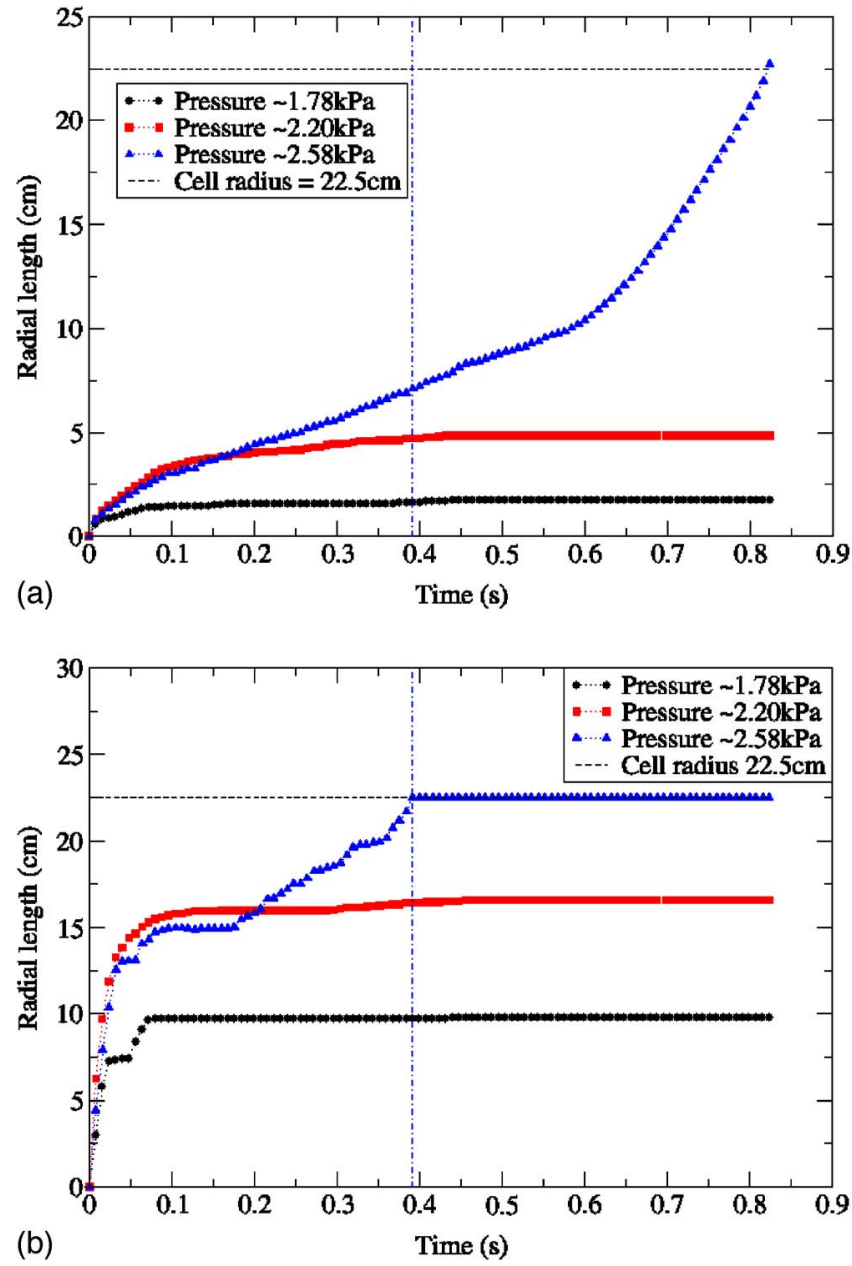

FIG. 13. (Color online) Experimental measurements of $r_{i}$ (a) and the maximum radial extent of the compacted region (b) for three different values of the injection pressures. The vertical line indicates the time when the compacted zone reaches the open boundary.

tation of an upper limit to the possible compaction in the already compacted zone (solid fractions are always below unity).

When the region where the packing can be compacted no further has reached the open boundary, the particles will be pushed out of the system. The (in-plane) friction is then reduced due to the smaller contact area with the confining walls. This situation is unstable and the propagating finger(s) accelerates. This is seen in Figs. 13(a) and 13(b): When the compacted zone reaches the outer boundary the most advanced finger, which is measured by $r_{i}$, accelerates. This is evident by the fact that the figure shows $d^{2} r_{i}(t) / d t^{2}>0$. Figure 14, which displays a comparison between an experiment and simulation at $\Delta P=3.00 \mathrm{kPa}$, shows the same effect. At the time $\sim 0.15 \mathrm{~s}$, indicated with a vertical blue line, the compacted zone reaches the boundary. From this moment the advancing finger starts to accelerate and the finger finally breaks through the model at the end of the measurement.

Another interesting feature is the decompaction zone, measured by $r_{d}$, near the boundary in the preferred growth direction. As the particles near the boundary start to feel the

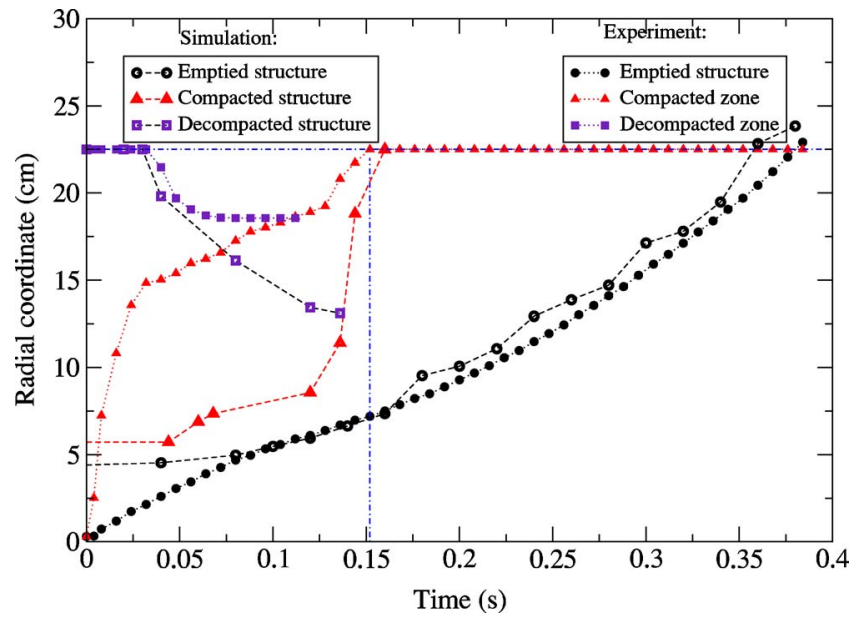

FIG. 14. (Color online) Experimental and simulation measurements of $r_{i}, r_{c}$, and $r_{d}$ at a pressure of approximately $3.00 \mathrm{kPa}$. The dashed vertical line at $\sim 0.15 \mathrm{~s}$ indicates the time for which the compacted zone reaches the open boundary.

pressure gradient, they are displaced and some of the outermost particles are pushed out of the cell. As can be seen from Fig. 14, the decompacted zone grows deeper into the material towards the center, in the opposite direction of air flow. While the decompacted zone saturates, the compacted region still grows along with the developing emptied structure. The compacted region eventually catches up with the decompacted zone, and starts to compact this region as well. Bubbles are observed near the external boundary in some experiments and appear to be coupled to this decompaction zone. The reduction of the granular density there will allow bubbles to form, as was observed in the simulations. In Fig. 14 there is good agreement between experiments and simulations for $r_{i}$ measurements, and qualitative agreement for the extension of the compacted regions and $r_{d}$.

Figure 15 shows a compacted structure after injection: The compacted (region 2) solid fraction is $\rho_{c}$ while the unperturbed zone (region 3 ) has kept the initial solid fraction $\rho_{0}$. By conservation of grain mass, we may write

$$
\rho_{0}\left(A_{1}+A_{2}\right)=\rho_{c} A_{2},
$$

where the areas $A_{1}$ and $A_{2}$ are shown in Fig. 15, and define

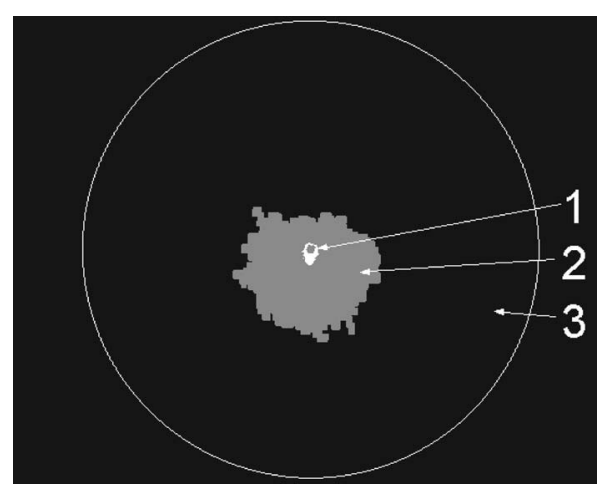

FIG. 15. Empty (1), compacted (2), and unperturbed regions (3) after air injection. 


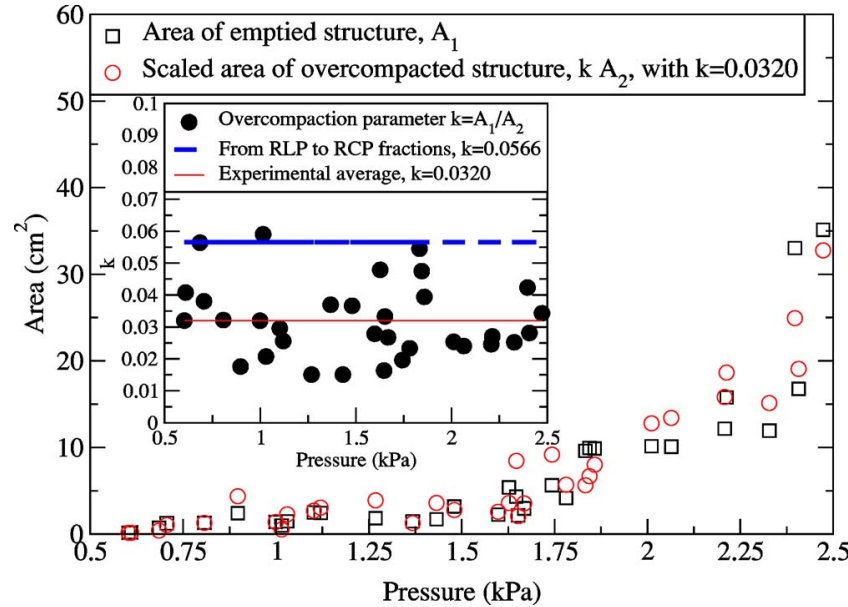

FIG. 16. (Color online) The estimated and experimentally measured area of the empty region as a function of pressure. The inset shows the compaction parameter $\mathrm{k}$ for our data set. The blue line shows an upper bound for $k$.

$$
k \equiv \frac{A_{1}}{A_{2}}=\frac{\Delta \rho_{s}}{\rho_{0}},
$$

where $\Delta \rho_{s}=\rho_{c}-\rho_{0}$. For a number of experiments performed at different injection pressures we have measured $A_{1}$ and $A_{2}$ by image analysis. The area of the injection hole is not included in the measured $A_{1}$ and $A_{2}$. In the inset of Fig. 16, $k=A_{1} / A_{2}$ is plotted as function of the pressure. The mean value of these fractions is found to be $\bar{k}=0.032 \pm 0.015$. An upper bound to this compaction parameter $k$ can in principle be estimated as follows: Studying the random loose packing (RLP) and random close packing (RCP) of spheres, Scott found $\rho_{\mathrm{RCP}}=0.635 \pm 0.005$ and $\rho_{\mathrm{RLP}}=0.601 \pm 0.005$ [68]. These are the lowest and highest densities for random packings of spheres-called, respectively, loose packed and close packed fractions. A transition between these two extreme random packing fractions would lead to the compaction parameter $k_{\max }=\Delta \rho_{s} / \rho_{0}=(0.635-0.601) / 0.601 \approx 0.056$. This value should correspond to the maximum $k$ obtained for a variation from the loosest to the most compact random packing. This upper bound is shown in the inset of Fig. 16.

By weighing the granular material, and measuring the area it covers in the cell, knowing the glass density, $2.6 \mathrm{~g} \mathrm{~cm}^{-3}$, we obtain the solid fraction 0.44 before air injection. Using Eq. (9) and the mean fraction $A_{1} / A_{2}=0.032$ found from experiments, the solid fraction is calculated to be $\rho_{c}=0.46$, which is $27 \%$ lower than the random close packed fraction $\rho_{\mathrm{RCP}}=0.635 \pm 0.005$. The solid fraction of the initial state is $26 \%$ lower than the random loose packed fraction $\rho_{\text {RLP }}=0.601 \pm 0.005$. These low solid fractions are consistent with the presence of the walls. The particles within the packing, which is only ten particle diameters thick, will feel the walls which cause a frustrated system with higher porosity for both the initial and the compacted state [71].

Given the average value of $k$ we can work backwards and calculate the area $A_{1}$ of the empty region from the area of the compacted region using as $A_{1}=k A_{2}$. Comparing this estimate

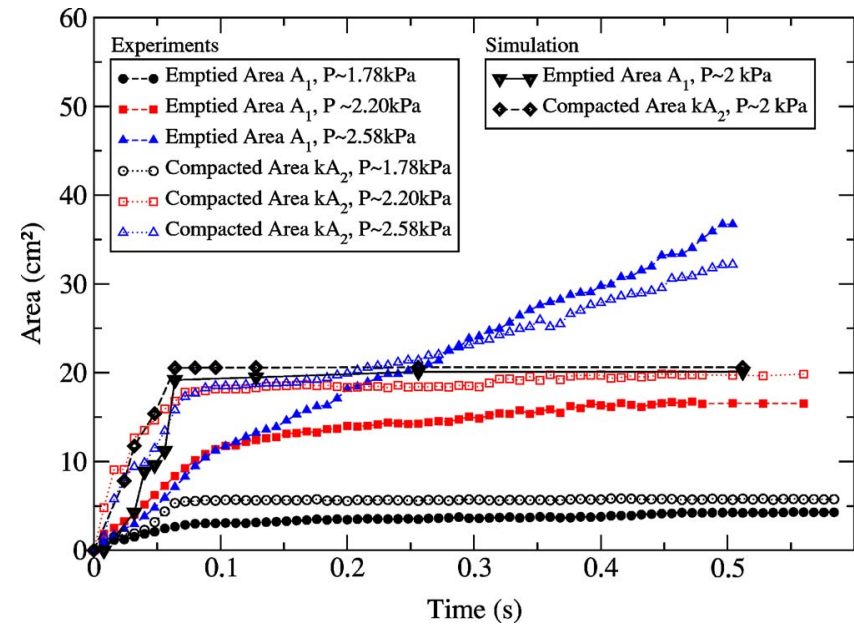

FIG. 17. (Color online) Area of the central emptied region and scaled compacted region for different injection pressures, in three experiments and one simulation.

of $A_{1}$ with measurements gives another way of viewing the fluctuations in $k$. Figure 16 shows this comparison using $k$ $=\Delta \rho_{s} / \rho_{0}=0.032$, which gives a good fit to the experimental results.

Figure 17 shows the measured area $A_{1}$ from three experiments (one for each of pressure regime 2, 3, and 4) and one simulation (regime two) as function of time. In this figure we have also plotted $k A_{2}$ where the $A_{2}$ are measured in the same experiments.

The first pressure regime structure is typically established after $\sim 0.1 \mathrm{~s}$ and can either stop evolving completely or grow slightly more in a slow fashion for a couple of tenths of seconds. In the second pressure regime, $A_{1}$ saturates after $0.45 \mathrm{~s}$. In the third regime we have the onset of instability. The measured area of both the branched empty region and the compacted region is larger. The outer perimeter of the compacted region stays uniform but asymmetric around the center, in accordance with the asymmetric shape of the emptied structure. The time for which the patterns are established and the final size and shape obtained are more or less the same as in the second regime; i.e., $\sim 1 \mathrm{~s}$. There might also here be some relatively slow "creeping" motion a couple of tenths of seconds after the structure is established. The compacted zone can reach all the way up to the open boundary, but with only a tiny amount of particles being pushed out of the cell and without the breakthrough instability setting in.

From the moment at which the flux of particles leaving the cell becomes continuous, the empty region expands rapidly. We see that the breakpoint where the structure growth slows down or reaches a plateau coincides for all three experimental cases and the simulation at about $t \approx 0.1 \mathrm{~s}$. The ripples in the experimental plots in Fig. 17 reflect the uncertainty related to the method of identifying and finding the areas.

Simulations and experiments reflect the same physical behavior in the sense that the different regimes as function of pressure are observed and that their evolution happens over the same times. The linear extent of the compacted or decompacted zones coincide reasonably well, as well as the 
area of the emptied and compacted zone. Some discrepancies for the magnitude of the area are present between numerics and experiments. These discrepancies are believed to arise from the subjective choice of threshold values used to define compaction: In the experimental measures, this threshold is hardly quantifiable, being related to the sensitivity of the camera used-since the compaction area is extracted by image treatment from subtraction images, as is illustrated in Fig. 3. In the numerical case, the value of the compacted area is rather sensitive to the adopted value for the threshold.

There are also discrepancies between the experimental emptied area and the calculated rescaled compacted area, which might be associated with the fact that an average $k$-value is used when estimating the area. From the inset in Fig. 16, we see that the $k$-values are spread quite wide around the average, and therefore might not always fit a specific set of data perfectly.

\section{SUMMARY AND CONCLUSIONS}

Studying the system experimentally and numerically, we have described the displacement behavior and patterns in granular materials confined in circular Hele-Shaw cellsqualitatively as well as quantitatively. Simulations have been a central tool to understand the system.

The driving force of this system is the pressure gradient over the material between the front of the pattern and the open boundary. The friction between the granular material and the confining plates act as the stabilizing mechanism. These two competing mechanisms may be controlled to produce a range of patterns resulting from air injection. Four well-defined pressure regimes giving different patterns have been found. In the first regime, no displacement of grains is recorded, so that the injected air just permeates through the pores of the granular material. In the second regime, particles are displaced and a roughly circular pattern appears. The structures become larger as the injection pressure is increased. The third regime is entered with the onset of instability as the shape of the displacement front loses its circular symmetry. This happens as the front starts to bulge and to form branches. The length of the branches increases with increasing injection pressure. As in the Saffmann-Taylor instability, the driving pressure gradient is larger ahead of the most developed finger. Hence, the most developed finger grows at the expense of the others.

For both the second and third pressure regimes, image analysis reveals a zone ahead of the emptied formation where the granular material becomes more dense. By grain conservation, we relate the area of the structure empty of grains $\left(A_{1}\right)$ and the area of the compacted region $\left(A_{2}\right)$. At pressures at which the compacted region reaches the boundary and particles are expelled, the in-plane friction will decrease. When a sufficient amount of particles is pushed out, such that the friction no longer can balance the hydrodynamic drag, the material will fluidize locally ahead of the most advanced finger, which eventually leads to breakthrough. At this point we find ourselves in the fourth pressure regime.

As an extension, system size and boundary condition dependences should be subject to both experimental and numerical investigations. It would also be interesting to examine the displacement field of the bulk zone using correlation imaging velocimetry technique.

\section{ACKNOWLEDGMENTS}

The work was supported by NFR, the Norwegian Research Council through a Petromax, and a SUP grant. We thank Ernesto Altshuler, Alex Hansen, Jean Schmittbuhl, Anke Lindner, and Christophe Chevalier for many interesting and helpful discussions.
[1] H. S. Hele-Shaw, Nature (London) 58, 34 (1898).

[2] P. G. Saffman and G. I. Taylor, in Proc. R. Soc. London, Ser. A 245, 312 (1958).

[3] H. Darcy, Les Fontaines Publiques de la Ville de Dijon (Dalmont, Paris, 1856).

[4] J. F. Davidson and D. Harrison, Fluidization (Academic, New York, 1971).

[5] J. F. Davidson, Bubbles in Fluidized Beds (Kluwer Academic, New York, 1995), p. 197.

[6] K. Lim, J. Zhu, and J. Grace, Int. J. Multiphase Flow 21, 141 (1995).

[7] S. McNamara, E. G. Flekkøy, and K. J. Måløy, Phys. Rev. E 61, 4054 (2000).

[8] G. W. Baxter, R. P. Behringer, T. Fagert, and G. A. Johnson, Phys. Rev. Lett. 62, 2825 (1989).

[9] K. J. Måløy, T. L. Pennec, E. G. Flekkøy, D. Bideau, M. Ammi, J. C. Messager, X. L. Wu, and A. Hansen, Physics of Dry Granular Media, NATO Advanced Studies Instituto, Series E: Applied Sciences, 350 (Kluwer Academic, Dordrecht, Boston, London, 1998).
[10] T. L. Pennec, K. J. Måløy, E. G. Flekkøy, J. C. Messager, and M. Ammi, Phys. Fluids 10, 3072 (1998).

[11] E. G. Flekkøy and K. J. Måløy, Phys. Rev. E 57, 6962 (1998).

[12] D. Gendron, H. Troadec, K. J. Måløy, and E. G. Flekkøy, Phys. Rev. E 64, 021509 (2001).

[13] E. G. Flekkøy, S. McNamara, K. J. Måløy, and D. Gendron, Phys. Rev. Lett. 87, 134302 (2001).

[14] X. I. Wu, K. J. Måløy, A. Hansen, M. Ammi, and D. Bideau, Phys. Rev. Lett. 71, 1363 (1993).

[15] T. L. Pennec, K. J. Måløy, A. Hansen, M. Ammi, D. Bideau, and X. I. Wu, Phys. Rev. E 53, 2257 (1996).

[16] E. Lemaire, Y. O. M. Abdelhaye, J. Larue, R. Benoit, P. Levitz, and H. van Damme, Fractals 1, 968 (1993).

[17] P. Mills, P. Cerasi, and S. Fautrat, Europhys. Lett. 29, 215 (1995).

[18] P. Cerasi and P. Mills, Phys. Rev. E 58, 6051 (1998).

[19] R. Chandler, J. Koplik, K. Lerman, and J. F. Willemsen, J. Fluid Mech. 119, 249 (1982).

[20] R. Lenormand and C. Zarcone, Phys. Rev. Lett. 54, 2226 (1985). 
[21] R. Lenormand and C. Zarcone, Transp. Porous Media 4, 599 (1989).

[22] G. M. Homsy, Annu. Rev. Fluid Mech. 19, 271 (1987).

[23] K. J. Måløy, J. Feder, and T. Jøssang, Phys. Rev. Lett. 55, 2688 (1985).

[24] O. I. Frette, K. J. Måløy, J. Schmittbuhl, and A. Hansen, Phys. Rev. E 55, 2969 (1997).

[25] E. Aker, K. J. Måløy, and A. Hansen, Phys. Rev. Lett. 84, 4589 (2000).

[26] G. Lovoll, Y. Meheust, R. Toussaint, J. Schmittbuhl, and K. J. Måløy, Phys. Rev. E 70, 026301 (2004).

[27] R. Toussaint, G. Lovoll, Y. Meheust, K. J. Måløy, and J. Schmittbuhl, Europhys. Lett. 71, 583 (2005).

[28] T. A. Witten and L. M. Sander, Phys. Rev. Lett. 47, 1400 (1981).

[29] T. A. Witten and L. M. Sander, Phys. Rev. B 27, 5686 (1983).

[30] P. Meakin, Phys. Rev. A 27, 1495 (1983).

[31] L. Paterson, Phys. Rev. Lett. 52, 1621 (1984).

[32] E. L. Hinrichsen, K. J. Måløy, J. Feder, and T. Jossang, J. Phys. A 22, L271 (1989).

[33] F. A. L. Dullien, Chem. Eng. Technol. 11, 407 (1988).

[34] J. Bear, Dynamics of Fluids in Porous Media (American Elsevier, New York, 1972).

[35] M. Sahimi, Rev. Mod. Phys. 65, 1393 (1993).

[36] P. van Meurs, Trans. Am. Inst. Min., Metall. Pet. Eng. 210, 295 (1957).

[37] A. K. Gustensen and D. H. Rothman, J. Geophys. Res. 98, 6431 (1993).

[38] H. Tchelepi and F. Orr, SPE Reservoir Eng. 9, 266 (1994).

[39] I. Ginzburg and P. Adler, Transp. Porous Media 20, 37 (1995).

[40] D. Bensimon, L. P. Kadanoff, S. Liang, B. I. Shraiman, and C. Tang, Rev. Mod. Phys. 58, 977 (1986).

[41] R. L. Chuoke, P. van Meurs, and C. van der Poel, Trans. Am. Inst. Min., Metall. Pet. Eng. 216, 188 (1959).

[42] J. V. Maher, Phys. Rev. Lett. 54, 1498 (1985).

[43] D. Bonn, H. Kellay, M. Ben Amar, and J. Meunier, Phys. Rev. Lett. 75, 2132 (1995).

[44] A. Lindner, D. Bonn, E. Poiré, M. B. Amar, and J. Meunier, J. Fluid Mech. 462, 237 (2002).

[45] G. Daccord, J. Nittmann, and H. E. Stanley, Phys. Rev. Lett. 56, 336 (1986).

[46] J. Nittman, G. Daccord, and H. E. Stanley, Nature (London) 314, 141 (1985).

[47] E. Lemaire, P. Levitz, G. Daccord, and H. van Damme, Phys. Rev. Lett. 67, 2009 (1991).

[48] H. Zhao and J. V. Maher, Phys. Rev. E 47, 4278 (1993).
[49] D. Mader, Hydraulic Proppant Fracturing and Gravel Packing (Elsevier, Amsterdam, 1989).

[50] Y. Gordeyev, Int. J. Rock Mech. Min. Sci. 30, 233 (1993).

[51] P. Valkø and M. J. Econimedes, Hydraulic Fracture Mechanics (John Wiley, New York, 1995).

[52] E. Fjaer, R. M. Holt, P. Horsrud, A. M. Raaen, and R. Risnes, Petroleum Related Rock Mechanics (Elsevier Science, New York, 1992).

[53] W. Fyfe, N. Price, and A. Thompson, Fluids in the Earth's Crust (Elsevier, Amsterdam, 1978).

[54] A. Levermann and I. Procaccia, Phys. Rev. Lett. 89, 234501 (2002)

[55] E. G. Flekkøy, A. Malthe-Sorenssen, and B. Jamtveit, J. Geophys. Res. 107, 2115 (2002).

[56] F. Tzschichholz, H. J. Herrmann, H. Roman, and M. Pfuff, Phys. Rev. B 49, 7056 (1994).

[57] F. Tzschichholz and H. Herrmann, Phys. Rev. E 51, 1961 (1995).

[58] H. A. Janssen, Zeitschrift des Vereines Deutscher Ingenieure 39, 1045 (1892).

[59] D. Gidaspau, Multiphase Flow and Fluidization (Academic, San Diego, 1994).

[60] P. Carman, Trans. Inst. Chem. Eng. 15, 150 (1937).

[61] J. P. Bardet, Introduction to Computational Granular Mechanic (Springer, Berlin, 1998), pp. 99-169.

[62] J. Christoffersen, M. Mehrabadi, and S. Nemat-Nasser, ASME J. Appl. Mech. 48, 339 (1981).

[63] J. D. Goddard, J. Non-Newtonian Fluid Mech. 2, 169 (1977).

[64] M. Allen and D. Tildesley, Computer Simulation of Liquids (Clarendon Press, Oxford, 1987).

[65] L. Brendel and S. Dippel, Physics of Dry Granular Media (Kluwer Academic, Dordrecht, 1998), p. 313.

[66] F. Radjai and D. Wolf, Granular Matter 1, 3 (1998).

[67] C. S. O'Hern, S. A. Langer, A. J. Liu, and S. R. Nagel, Phys. Rev. Lett. 88, 075507 (2002).

[68] G. D. Scott, Nature (London) 188, 908 (1960).

[69] J. Duran, Sands, Powders and Grains: An Introduction to the Physics of Granular Materials (Springer, Berlin, 2000).

[70] A. Atman, P. Brunet, J. Geng, G. Reydellet, G. Combe, P. Claudin, R. Behringer, and E. Clement, J. Phys.: Condens. Matter 17, S2391 (2005).

[71] D. R. Nelson, in Topological Disorder in Condensed Matter: Proceedings of the Fifth Taniguchi International Symposium, edited by F. Yonesawa and T. Ninomiya (Springer, Berlin, 1983), pp. 164-180. 